\title{
Microwave assisted sulphated titania catalyzed aldol condensation: Synthesis of some (E) 3-(2-naphthyl)- 1-(substituted phenyl)-2-propen-1-ones under solvent-free conditions
}

\author{
P. Janaki ${ }^{1}$, K. G. Sekar ${ }^{1, *}$, G. Thirunarayanan ${ }^{2}$ \\ ${ }^{1}$ Department of Chemistry, National College, Tiruchirappalli - 620 001, India \\ ${ }^{2}$ Department of Chemistry, Annamalai University, Annamalainagar - 608 002, India \\ *E-mail address: drkgsekar@yahoo.co.in
}

\begin{abstract}
A series of titled chalcones were synthesized by aldol condensation between 2-naphthaldehyde and various substituted acetophenones using solid acidic solid sulphated titania catalyst under microwave irradiation. The yields of these chalcones are more than $90 \%$. The synthesized chalcones are characterized by their physical constants, analytical and spectroscopic data.
\end{abstract}

Keywords: Aldol condensation; Sulphated titania, Microwave irradiation; 2-propen-1-ones

\section{INTRODUCTION}

Chalcones or $\alpha, \beta$-unsaturated ketones are enones [1]. They possess vinyl and carbonyl group adjacently and aryl or alkyl group in both of the ends [2]. They exist as $E$ s-cis and strans conformers [3]. These conformers were confirmed by IR and NMR spectra [4]. Presence of vinyl, carbonyl and the polar substituents in the aryl rings of chalcones shows an important biological activities [5] such as antimicrobial [6], antidepressants [7], antiplosmodial [8], anti-aids [9] and insect antifeedant activities [10,11].

Various solvent-free and solvent assited methods are available for synthesis of chalcones. Many catalysts were applied for synthesis of chalcones using either solvent assisted or solvent-free methods such as EtOH-NaOH [12], MeOH-KOH [13], EtOH-KOH [14], $\mathrm{MgCl}_{2}$ [15], silica-sulphuric acid [16], anhydrous zinc chloride [17], clay [18], Hydrotalcite [19], ground chemistry catalysts-grinding the reactants with sodium hydroxide [10], aqueous alkali in lower temperature [20], solid sulphonic acid from bamboo [21], barium hydroxide [22] anhydrous sodium bicarbonate [23], microwave assisted synthesis [24], fly-ash:water [25], fly-ash: $\mathrm{H}_{2} \mathrm{SO}_{4}$ [26], fly-ash:PTS [27], $\mathrm{NaOH}-\mathrm{CTABr}$ [11], $\mathrm{SiO}_{2}-$ $\mathrm{H}_{3} \mathrm{PO}_{4}[28], \mathrm{SOCl}_{2}[29]$ and sulfated titania [30].

Within the above view, there is no report available for synthesis of titled compound by sulphated titania assisted aldol condensation of 2-naphthaldehyde and substituted acetophenones under microwave irradiation. 
Therefore the authors have taken efforts for solvent-free synthesis of titled compounds and recorded their IR and NMR spectra for characterization.

\section{EXPERIMENTAL}

\section{1. General}

All chemicals used were purchased from Sigma-Aldrich and E-Merck chemical companies. Melting points of all chalcones were determined in open glass capillaries on SUNTEX melting point apparatus and are uncorrected. The UV spectra of all synthesized chalcones were recorded in ELICO-BL222 SPECTROMETER $\left(\lambda_{\max } \mathrm{nm}\right)$ in spectral grade methanol.

Infrared spectra $\left(\mathrm{KBr}, 4000-400 \mathrm{~cm}^{-1}\right)$ were recorded on AVATAR-300 Fourier transform spectrophotometer. Bruker AV400 NMR spectrometer has been used for recording NMR spectra, operating at $400 \mathrm{MHz}$ has been utilized for recording ${ }^{1} \mathrm{H}$ spectra and $100 \mathrm{MHz}$ for ${ }^{13} \mathrm{C}$ spectra in $\mathrm{CDCl}_{3}$ solvent using TMS as internal standard. Mass spectra were recorded on a SIMADZU GC-MS2010 Spectrometer using Electron Impact (EI) techniques.

\section{2. General procedure for synthesis of (E)-3-(2-naphthyl)-1-(substituted phenyl)-2- propen-1-ones}

Appropriate quantities of substituted acetophenones $(2 \mathrm{mmol})$ and 2-naphthaldehyde (2 mmol) and $0.15 \mathrm{~g}$ of sulfated titania were taken in a $50 \mathrm{~mL}$ beaker and closed with the lid. This mixture was subjected to microwave irradiation for 2-4 minutes at $650 \mathrm{~W}$ (Scheme 1) (Samsung, Microwave Oven, 100-700 W).

After completion of the reaction, dichloromethane $(20 \mathrm{~mL})$ was added, followed by simple filtration. The filtrate was concentrated and the obtained solid was purified by recrystallization. The synthesized chalcones were characterized by their physical constants, $\mathrm{UV}, \mathrm{IR},{ }^{1} \mathrm{H}$ and ${ }^{13} \mathrm{C}$ NMR and Mass spectral data. Analytical and Mass spectral data are presented in Table 1.

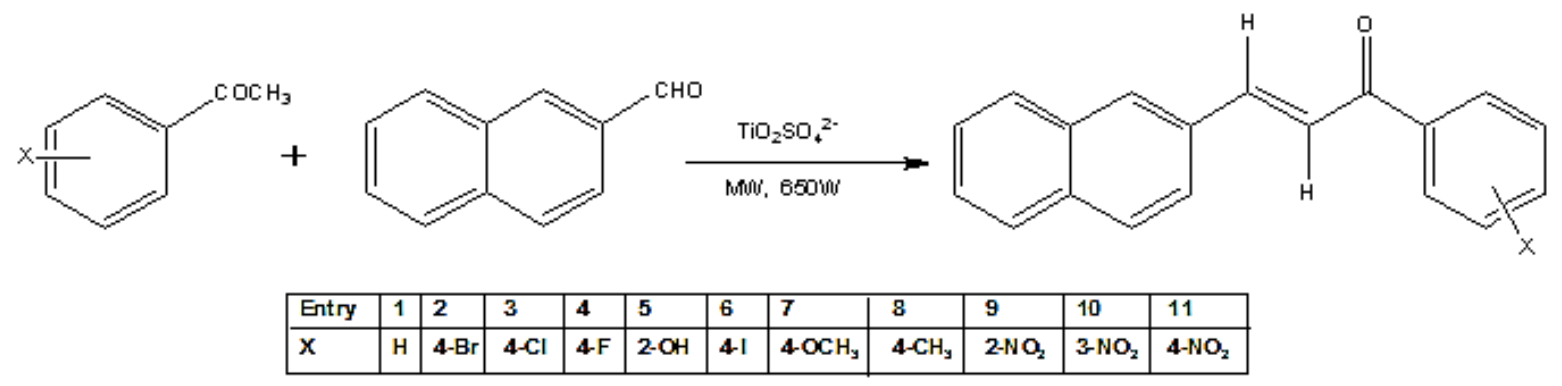

Scheme 1. Synthesis of (E)-3-(2-naphthyl)-1-(substituted phenyl)-2-propen-1-ones. 
Table 1. Physical constants, analytical and mass fragments (m/z) data of (E)-3-(2-naphthyl)-1(substituted phenyl)-2-propen-1-ones.

\begin{tabular}{|c|c|c|c|c|c|c|}
\hline Entry & $\mathbf{X}$ & M.F. & $\begin{array}{c}\text { M.W } \\
.\end{array}$ & $\begin{array}{l}\text { Yield } \\
(\%)\end{array}$ & $\begin{array}{l}\text { m.p. } \\
(\circ \mathrm{C})\end{array}$ & $\begin{array}{l}\text { Mass } \\
(\mathbf{m} / \mathbf{z})\end{array}$ \\
\hline 1 & $\mathrm{H}$ & $\mathrm{C}_{19} \mathrm{H}_{14} \mathrm{O}$ & 258 & 94 & $\begin{array}{c}111-112 \\
(111-112)^{31}\end{array}$ & $258\left[\mathrm{M}^{+}\right]$ \\
\hline 2 & $4-\mathrm{Br}$ & $\mathrm{C}_{19} \mathrm{H}_{13} \mathrm{BrO}$ & 337 & 91 & $98-99(96-98)^{31}$ & $\begin{array}{l}337\left[\mathrm{M}^{+}\right], \\
339\left[\mathrm{M}^{2+}\right]\end{array}$ \\
\hline 3 & $4-\mathrm{Cl}$ & $\mathrm{C}_{19} \mathrm{H}_{13} \mathrm{ClO}$ & 219 & 92 & $\begin{array}{c}116-117 \\
(116-117)^{32}\end{array}$ & $\begin{array}{l}219\left[\mathrm{M}^{+}\right], \\
221\left[\mathrm{M}^{2+}\right]\end{array}$ \\
\hline 4 & $4-\mathrm{F}$ & $\mathrm{C}_{19} \mathrm{H}_{13} \mathrm{FO}$ & 276 & 90 & $\begin{array}{c}105-106 \\
(105-106)^{32}\end{array}$ & $\begin{array}{l}276\left[\mathrm{M}^{+}\right], \\
278\left[\mathrm{M}^{2+}\right]\end{array}$ \\
\hline 5 & $2-\mathrm{OH}$ & $\mathrm{C}_{19} \mathrm{H}_{14} \mathrm{O}_{2}$ & 274 & 93 & $88-89$ & $274\left[\mathrm{M}^{+}\right]$ \\
\hline 6 & $4-\mathrm{I}$ & $\mathrm{C}_{19} \mathrm{H}_{13} \mathrm{IO}$ & 384 & 92 & $\begin{array}{c}118-119 \\
(115-116)^{33}\end{array}$ & $\begin{array}{l}384\left[\mathrm{M}^{+}\right], \\
386\left[\mathrm{M}^{2+}\right]\end{array}$ \\
\hline 7 & $4-\mathrm{OCH}_{3}$ & $\mathrm{C}_{20} \mathrm{H}_{16} \mathrm{O}_{2}$ & 288 & 95 & $122-123$ & $288\left[\mathrm{M}^{+}\right]$ \\
\hline 8 & $4-\mathrm{CH}_{3}$ & $\mathrm{C}_{20} \mathrm{H}_{16} \mathrm{O}$ & 272 & 90 & $112-113$ & $272\left[\mathrm{M}^{+}\right]$ \\
\hline 9 & $2-\mathrm{NO}_{2}$ & $\mathrm{C}_{19} \mathrm{H}_{13} \mathrm{NO}_{3}$ & 303 & 90 & $132-133$ & $303\left[\mathrm{M}^{+}\right]$ \\
\hline 10 & $3-\mathrm{NO}_{2}$ & $\mathrm{C}_{19} \mathrm{H}_{13} \mathrm{NO}_{3}$ & 303 & 90 & $127-128$ & $303\left[\mathrm{M}^{+}\right]$ \\
\hline 11 & $4-\mathrm{NO}_{2}$ & $\mathrm{C}_{19} \mathrm{H}_{13} \mathrm{NO}_{3}$ & 303 & 90 & $\begin{array}{c}119-120 \\
(199-122)^{33}\end{array}$ & $303\left[\mathrm{M}^{+}\right]$ \\
\hline
\end{tabular}

\section{RESULTS AND DISCUSSION}

In our organic chemistry research laboratory, we attempts to synthesize aryl chalcone derivatives by crossed-aldol condensation of electron withdrawing as well as electron donating group substituted acetophenone and benzaldehydes in the presence of vigorous acidic catalyst sulphated titania in microwave irradiation.

Hence the authors have synthesized the chalcone derivatives by the reaction between 2 mmol of 2-naphthaldehyde, $2 \mathrm{mmol}$ substituted acetophenones in microwave irradiation with $0.15 \mathrm{~g}$ of sulphated titania catalyst (Scheme 1).

During the course of this reaction sulphated titania catalyzes aldol-condensation between aryl ketone and aldehydes and elimination of water gave the chalcones. The yields of the chalcones in this reaction are more than $90 \%$.

This condensation follows acid catalyzed mechanism. The spectroscopic data of synthesized chalcones are presented in Tables 2 and 3. These data are supported for the formation of chalcones [34-47]. 
Table 2. Infrared spectral data $\left(v, \mathrm{~cm}^{-1}\right)$ of $(E)-3$-(2-naphthyl)-1-(substituted phenyl)-2-propen-1-ones.

\begin{tabular}{|c|c|c|c|c|c|c|c|}
\hline Entry & $\mathbf{X}$ & $\mathbf{C O}$ \\
s-cis & $\mathbf{C O}$ & s-trans & $\mathbf{C H}_{i p}$ & $\mathbf{C H}_{\boldsymbol{p}}$ & $\mathbf{C H}=\mathbf{C H}_{\boldsymbol{~} \boldsymbol{p}}$ & $\mathbf{C =}_{\boldsymbol{o p}}$ \\
\hline 1 & $\mathrm{H}$ & 1659.82 & 1602.30 & 1209.31 & 702.37 & 1087.08 & 688.76 \\
\hline 2 & $4-\mathrm{Br}$ & 1679.91 & 1634.24 & 1212.20 & 750.28 & 1017.35 & 691.86 \\
\hline 3 & $4-\mathrm{Cl}$ & 1659.39 & 1598.43 & 1206.09 & 814.40 & 1012.04 & 687.68 \\
\hline 4 & $4-\mathrm{F}$ & 1661.55 & 1602.11 & 1207.88 & 781.53 & 1018.27 & 693.75 \\
\hline 5 & $2-\mathrm{OH}$ & 1654.64 & 1602.11 & 1258.11 & 739.99 & 1074.79 & 685.65 \\
\hline 6 & $4-\mathrm{I}$ & 1661.28 & 1609.06 & 1185.52 & 729.60 & 1027.43 & 683.13 \\
\hline 7 & $4-\mathrm{OCH}_{3}$ & 1658.91 & 1604.11 & 1206.97 & 749.59 & 1029.51 & 673.24 \\
\hline 8 & $4-\mathrm{CH}_{3}$ & 1658.33 & 1600.33 & 1209.14 & 731.63 & 1010.58 & 677.15 \\
\hline 9 & $2-\mathrm{NO}_{2}$ & 1658.59 & 1592.57 & 1176.17 & 816.30 & 1007.20 & 674.17 \\
\hline 10 & $3-\mathrm{NO}_{2}$ & 1677.41 & 1634.21 & 1161.21 & 876.74 & 1017.14 & 655.16 \\
\hline 11 & $4-\mathrm{NO}_{2}$ & 1678.54 & 1632.41 & 1148.24 & 868.32 & 1056.82 & 673.26 \\
\hline
\end{tabular}

Table 3. NMR spectral data ( $\delta$, ppm) of (E)-3-(2-naphthyl)-1-(substituted phenyl)-2-propen-1-ones.

\begin{tabular}{|c|c|c|c|c|c|c|}
\hline Entry & $\mathbf{X}$ & $\mathbf{H}_{\boldsymbol{\alpha}}$ & $\mathbf{H}_{\boldsymbol{\beta}}$ & $\mathbf{C O}$ & $\mathbf{C}_{\boldsymbol{\alpha}}$ & $\mathbf{C}_{\boldsymbol{\beta}}$ \\
\hline $\mathbf{1}$ & $\mathrm{H}$ & 7.622 & 8.936 & 188.85 & 123.64 & 145.19 \\
\hline $\mathbf{2}$ & $4-\mathrm{Br}$ & 7.002 & 7.958 & 188.56 & 125.55 & 136.90 \\
\hline $\mathbf{3}$ & $4-\mathrm{Cl}$ & 7.843 & 8.057 & 189.04 & 123.49 & 134.71 \\
\hline $\mathbf{4}$ & $4-\mathrm{F}$ & 7.657 & 8.075 & 188.00 & 123.32 & 134.18 \\
\hline $\mathbf{5}$ & $2-\mathrm{OH}$ & 7.987 & 8.024 & 189.71 & 123.61 & 134.50 \\
\hline $\mathbf{6}$ & $4-\mathrm{I}$ & 7.437 & 7.878 & 188.17 & 124.75 & 139.65 \\
\hline $\mathbf{7}$ & $4-\mathrm{OCH}_{3}$ & 7.670 & 7.760 & 188.75 & 123.74 & 144.11 \\
\hline $\mathbf{8}$ & $4-\mathrm{CH}_{3}$ & 7.684 & 7.945 & 190.03 & 123.73 & 144.53 \\
\hline $\mathbf{9}$ & $2-\mathrm{NO}_{2}$ & 7.620 & 8.019 & 189.08 & 123.62 & 134.5 \\
\hline $\mathbf{1 0}$ & $3-\mathrm{NO}_{2}$ & 7.869 & 8.041 & 189.39 & 125.71 & 145.56 \\
\hline $\mathbf{1 1}$ & $4-\mathrm{NO}_{2}$ & 7.871 & 8.132 & 189.78 & 125.69 & 144.97 \\
\hline
\end{tabular}




\section{CONCLUSIONS}

About eleven titled chalcones were synthesized by aldol condensation between 2naphthaldehyde and various substituted acetophenones using solid acidic solid sulphated titania catalyst under microwave irradiation. The yields of these chalcones are more than 90 $\%$. The synthesized chalcones are characterized by their physical constants, analytical and spectroscopic data. These data are supported for the formation of chalcones.

\section{ACKNOWLEDGMENT}

The authors thank DST-NMR facility, Department of Chemistry, Annamalai University, Annamalainagar-608 002 for recording NMR spectra of all compounds.

\section{References}

[1] Ranganathan K., et al., International Letters of Chemistry, Physics and Astronomy 4 (2012) 66-75.

[2] Arulkumaran R., et al., IUP J. Chem. 3(1) (2010) 82-98.

[3] Arulkumaran R., Sundararajan R., Vijayakumar S., Sakthinathan S. P., Suresh R., Kamalakkannan D., Ranganathan K., Vanangamudi G., Thirunarayanan G., J. Saudhi Chem. Soc. 2012. Doi: 10.1016/j.jscs.2012.09.006

[4] Janaki P., Sekar K. G., Thirunarayanan G., J. Saudi Chem. Soc. 2013. Doi:10.1016/j.jscs.2012.11.013

[5] Thirunarayanan G., Vanangamudi G., Subramanian M., Org. Chem.: An Indian Journal 9(1) (2012) 1-16.

[6] Sivakumar M., Phrabu Sreeneivasan S., Kumar V., Doble M., Bioorg. Med. Chem. Lett. 17(10) (2007) 3169-3172.

[7] Liu X., Go M. L., Bioorg. Med. Chem. 14(1) (2006) 153-163.

[8] Arulkumaran R., Sundararajan R., Vanangamudi G., Subramanian M., Ravi K., Sathiyendidran V., Srinivasan S., Thirunarayanan G., IUP J. Chem. 3(1) (2010) 82-98.

[9] Deng J., Sanchez T., Lalith Q. A. M., Bioorg. Med. Chem. 15(14) (2007) 4985-5002.

[10] Thirunarayanan G., J. Indian Chem. Soc. 84 (2008) 447-451.

[11] Thirunarayanan G., Surya S., Srinivasan S., Vanangamudi G., Sathiyendiran V., Spectrochim. Acta 75A (2010) 152-156.

[12] Vanangamudi G., Subramanian S., Jayanthi P., Arulkumaran R., Kamalakkannan D., Thirunarayanan G., Arab. J. Chem., 201, DOI:10.1016/j.arabjc.2010.07.019.

[13] Tran T. D., Park H., Ecker G. F., Thai K. M., 3,2'-Hydroxychalcone Analogues: Synthesis and Structure-PGE2 Inhibitory Activity Relationship, $12^{\text {th }}$ International Electronic Conference on Synthetic Organic Chemistry (ECSOC-12) 1-30 November 2008; http://www.usc.es/congresos/ecsoc/12/ECSOC12.htm

$\&$ http://www.mdpi.org/ecsoc-12 No. C0011, pp 1-7. 
[18] Mohan S. B., Behera T. P., Ravi Kumar B. V. V., Int. J. ChemTech. Res. 2(3) (2010) 1634-1637.

[15] Orsini F., Sello G., Fumagalli T., Synlett. 2006, 1717-1718.

[16] Thirunarayanan G., Vanangamudi G., Arkivoc. 12 (2006) 58-64.

[17] Thirunarayanan G., Ananthakrishna Nadar P., J. Indian Chem. Soc. 83(11) (2006) 1107-1112.

[18] Ballini R., Bosica G., Maggi R., Ricciutelli M., Righi P., Sartori G., Sartorio R., Green Chem. 3 (2001) 178-180.

[19] Solhy A., Tahir R., Sebti S., Skouta R., Bousmina M., Zahouily M., Larzek M., Appl. Catal. A: General. 374 (2010) 189-193.

[20] Basaif S. A., Sobahi T. R., Khalil A. K., Hassan M. A., Bulletin of the Korean Chem. Soc. 26(11) (2005) 1677-1681.

[21] Xu Q., Yang Z., Yin D., Zhang F., Catal. Commun. 9(1) (2008) 1579-1582.

[22] Kumar P., Kumar S., Husain K., Kumar A., Bioorg. Med. Chem. 18(14) (2010) $4965-$ 4974.

[23] Zhang Z., Dong Y. W., Wang G. W., Chem. Lett. 32(10) (2003) 966-967.

[24] Thirunarayanan G., IUP. J. Chem. 3(4) (2010) 35-54.

[25] Thirunarayanan G., Elixir Org. Chem. 45 (2012) 7898-7905.

[26] Thirunarayanan G., Mayavel P., Thirumurthy K., Spectrochimica Acta. 91A (2012) 1822.

[27] Sundararajan R., Arulkumaran R., Vijayakumar S., Kamalakkannan D., Suresh R., Ranganathan K., Sakthinathan S. P., Vanangamudi G., Thirumurthy K., Mayavel P., Thirunarayanan G., Int. J. Pharm. Chem. Sci. 1(4) (2012) 1657-1677.

[28] Janaki P., Sekar K. G., Thirunarayanan G., Org. Chem: An Indian J. 9(2) (2013) 68-80.

[29] Arulkumaran R., et al., International Letters of Chemistry, Physics and Astronomy 4 (2012) 17-38.

[30] Sundararajan R., Q-science Connect, 2013.

DOI: http://dx.doi.org/10.5339/connect.2013.30

[31] Mitina V. G., Shenkoo A. O. D., Sukhorukov A. A., Lavrushin V. F., Teror. Eksp. Khim. 20(2) (1984) 147-150.

[32] Vibhute Y. B., J. Indian Chem. Soc. 53(7) (1976) 736-740.

[33] Dhar D. N., Singh R. K., J. Indian Chem. Soc. 49(3) (1972) 241-245.

[34] R. Arulkumaran, S. Vijayakumar, R. Sundararajan, S. P. Sakthinathan, D.

Kamalakkannan, R. Suresh, K. Ranganathan, P. R. Rajakumar, G. Vanangamudi, G.

Thirunarayanan, International Letters of Chemistry, Physics and Astronomy 5 (2013) 21-38.

[35] S. P. Sakthinathan, R. Suresh, V. Mala, K.Sathiyamoorthi, D. Kamalakkannan, K. Ranganathan, R. Arulkumaran, S. Vijayakumar, R. Sundararajan, G. Vanangamudi, G. Thirunarayanan, International Letters of Chemistry, Physics and Astronomy 6 (2013) 77-90. 
[36] K. Sathiyamoorthi, V. Mala, R. Suresh, S. P. Sakthinathan, D. Kamalakkannan, K. Ranganathan, R. Arulkumaran, R. Sundararajan, S. Vijayakumar, G. Vanangamudi, G. Thirunarayanan, International Letters of Chemistry, Physics and Astronomy 7(2) (2013) 102-119.

[37] G. Thirunarayanan, K. G. Sekar, International Letters of Chemistry, Physics and Astronomy 8(2) (2013) 160-174.

[38] K. G. Sekar, G. Thirunarayanan, International Letters of Chemistry, Physics and Astronomy 8(3) (2013) 249-258.

[39] S. Vijayakumar, R. Arulkumaran, R. Sundararajan, S. P. Sakthinathan, R. Suresh, D. Kamalakkannan, K. Ranganathan, K. Sathiyamoorthy, V. Mala, G. Vanangamudi, G. Thirunarayanan, International Letters of Chemistry, Physics and Astronomy 9(1) (2013) 68-86.

[40] G. Thirunarayanan, K. G. Sekar, International Letters of Chemistry, Physics and Astronomy 10(1) (2013) 18-34.

[41] R. Sundararajan, R. Arulkumaran, S. Vijayakumar, D. Kamalakkannan, R. Suresh, S. John Joseph, K. Ranganathan, S. P. Sakthinathan, G. Vanangamudi, G. Thirunarayanan, International Letters of Chemistry, Physics and Astronomy 1 (2014) 67-73.

[42] S. John Joseph, R. Arulkumaran, D. Kamalakkannan, S. P. Sakthinathan, R. Sundararajan, R. Suresh, S. Vijayakumar, K. Ranganathan, N. Kalyanasundaram, G. Vanangamudi, G. Thirunarayanan, International Letters of Chemistry, Physics and Astronomy 4 (2014) 48-65.

[43] G. Thirunarayanan, R. Sundararajan, R. Arulkumaran, International Letters of Chemistry, Physics and Astronomy 4 (2014) 82-97.

[44] G. Thirunarayanan, International Letters of Chemistry, Physics and Astronomy 4 (2014) 109-116.

[45] G. Thirunarayanan, International Letters of Chemistry, Physics and Astronomy 5 (2014) 89-98.

[46] S. John Joseph, D. Kamalakkannan, R. Arulkumaran, S. P. Sakthinathan, R. Suresh, R. Sundararajan, S. Vijayakumar, K. Ranganathan, G. Vanangamudi, G. Thirunarayanan, International Letters of Chemistry, Physics and Astronomy 5 (2014) 99-123.

[47] G. Thirunarayanan, S. Pazhamalai, K. G. Sekar, International Letters of Chemistry, Physics and Astronomy 8 (2014) 38-46. 